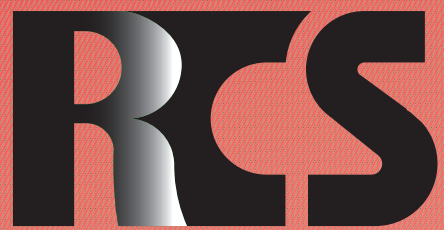

Depósito legal ppi $201502 Z U 4662$

Esta publicación científica en formato digital es continuidad de la revista impresa Depósito Legal: pp $197402 Z U 789$

- ISSN: 1315-9518 • ISSN-E: 2477-9431

Revista de Ciencias Sociales

Universidad del Zulia. Revista de la Facultad de Ciencias Económicas y Sociales Vol. XXVII. No. 2

Abril-Junio 2021

Esta publicación científica en formato digital es continuidad de la revista impresa Depósito Legal: pp $197402 Z$ Z789 ISSN: 1315-9518 


\title{
Programa de transición a la vida adulta en Andalucía: Percepción de profesionales y extutelados
}

\author{
Sevillano-Monje, Verónica* \\ Ballesteros-Moscosio, Miguel-Ángel** \\ González-Monteagudo, José
}

\section{Resumen}

Numerosas investigaciones internacionales demuestran que los jóvenes extutelados tienen mayores tasas de desempleo que su mismo grupo de edad. En este artículo se pretende conocer los beneficios para facilitar la inserción sociolaboral del programa español de Alta Intensidad Mayoría de Edad +18 a partir de las percepciones y experiencias de los jóvenes y sus orientadores. Este programa está destinado a jóvenes que salen del Sistema de Protección de menores al cumplir la mayoría de edad como alternativa tras la tutela. En este estudio descriptivo se hace uso de un diseño metodológico mixto, fijo y emergente, combinando las técnicas de entrevista y encuesta para la recogida de datos. La muestra la forman dos profesionales, seis jóvenes beneficiaros del programa y cuatro egresados, entre 18 y 23 años. Los resultados apuntan a la consideración del impacto positivo del programa, al reconocer todos los participantes su utilidad en la mejora de sus posibilidades de inserción sociolaboral. Se concluye, que el programa es necesario y positivo, siendo una oportunidad única para los jóvenes. No obstante, se desvelan también debilidades tales como la necesidad de una mayor implicación de la Administración, el aumento de subvenciones y concienciación de la existencia de estos programas.

Palabras clave: Transición a la vida adulta; jóvenes extutelados; Programas de transición; inserción laboral; emancipación.

\footnotetext{
* Doctoranda en Educación. Máster en Formación y Orientación Profesional para el Empleo. Pedagoga. Docente e Investigadora en Formación de la Universidad de Sevilla, España. E-mail: vsevillano@us.es iD ORCID: https://orcid.org/0000-0002-1533$\underline{5829}$

** Doctor en Educación. Pedagogo. Profesor Contratado Doctor de la Universidad de Sevilla, España. E-mail: miguelanba@us.es iD ORCID: https://orcid.org/0000-0001-9522-4303

*** Doctor en Educación. Pedagogo. Profesor Titular de la Universidad de Sevilla, España. E-mail: monteagu@us.es (iD) ORCID: https://orcid.org/0000-0002-3094-8092
}

Recibido: 2020-12-10 • Aceptado: 2021-02-27 


\title{
Programme of transition to adulthood in Andalusia: Perceptions of counsellors and care leavers
}

\begin{abstract}
Numerous international studies show that care leavers have higher unemployment rates than their age group. This article aims to find out about the benefits to facilitate the social and labour insertion of the Spanish programme of High Intensity Coming of Age +18 from the perceptions and experiences of young people and their counsellors. This program is aimed at young people care leavers from child welfare system when they reach the age of majority as an alternative after guardianship. This descriptive study uses a mixed, fixed and emergent methodological design, combining interview and survey techniques for data collection. The sample is made up of professionals, young people benefiting from the programme and graduates, aged between 18 and 23. The results point to the consideration of the positive impact of the program, as all the participants recognize its usefulness in improving their possibilities of social and labor insertion. It is concluded, that the programme is necessary and positive, being a unique opportunity for young people. However, weaknesses are also revealed such as the need for greater involvement of the Administration, the increase in subsidies and awareness of the existence of these programs.
\end{abstract}

Keywords: Transition to adulthood; care leavers, transition programmes, labour market insertion, emancipation.

\section{Introducción}

En el estado español, como dice el artículo 12 de la Constitución Española, "los españoles son mayores de edad a los 18 años" (Gobierno de España, Ministerio de la Presidencia, 1978). La mayoría de edad supone la adquisición de la plena capacidad de obrar de la persona, entendida como la aptitud para poder ejercer los derechos y obligaciones de la que es titular. La diferencia entre mayoría y minoría de edad legal, tiene como fin la protección de los intereses de la persona, y por eso despliega en su favor toda una serie de instituciones tuitivas de sus derechos y su patrimonio. Sin embargo, el Legislador considera que, a partir de una determinada edad -los 18 años en el caso español-, se le ha de presumir a la persona el suficiente autogobierno psíquico para ejercer por sí misma sus propios derechos.

No obstante, el proceso de madurez y desarrollo emocional, social y fisiológico no tiene por qué darse en el mismo momento. Es por este motivo que los menores del Sistema de Protección en España, al cumplir los 18 años, deben de abandonar los Centros de Protección de menores, para iniciar la vida adulta de manera autónoma.

En el año 2018, se dieron de baja por mayoría de edad en España, un total de 4.002 jóvenes (el 21\% de las bajas totales) del Sistema de Protección (Ministerio de Sanidad, Consumo y Bienestar Social, 2019). En la región de Andalucía, según el informe de evaluación de la Dirección General de Infancia y Familias, la cifra de jóvenes que salieron del Sistema de Protección por alcanzar la mayoría de edad no superó los 900.

Estos jóvenes tienen desventajas laborales, condicionadas por los factores educativos y dificultades en sus procesos de adaptación a los contextos sociales, escolares y laborales, por lo que tienen un escaso dominio de competencias, debido a factores de riesgo físico, psicosociales y contextuales (Jurado, 
Olmos y Pérez, 2015; Saldaña, et al., 2020; Coronel y Farje, 2020).

En esta línea, Gudžinskienè, Raudeliūnaitè y Uscila (2017) explican que estos jóvenes enfrentan numerosas dificultades por vivir en un centro de protección debido a la falta de habilidades de autoempleo y autoayuda. Además, su estudio reveló que presentan restricciones de habilidades personales, fundamentales para la vida independiente como la adecuada autoevaluación, autocontrol y autoconfianza.

Concretamente, las dificultades de acceso al mercado laboral de este colectivo son mucho mayores: Tienen menos posibilidades de ser empleados y ganan sustancialmente menos que los jóvenes de la población general que no han sido tutelados (Dworsky y Gitlow, 2017). Como consecuencia, el proceso de transición a la vida adulta es más acelerado y comprimido que los jóvenes de la misma franja de edad (Stein, 2006). Además, al cumplir los 18 años y tener que abandonar los centros de protección, es probable que queden desamparados, al no poder volver con sus familias, o no ser lo más conveniente este retorno (Martín, 2015).

Los datos de Eurostat (2020), indican que la edad media de salida del hogar parental en las familias de origen de los jóvenes europeos es de 26,3 años y la tasa de desempleo en la UE-28 para los jóvenes de 15 a 24 años es del 14,6\%. En España, los datos son, respectivamente, 29,5 años y $34,6 \%$. Según el Instituto Nacional de Estadística (INE, 2020), a finales de 2019, la tasa de desempleo entre los jóvenes de 16 a 19 años es muy elevada $(43,9 \%)$, mientras que esa tasa de desempleo de los 20 a los 24 años es más baja (27,72\%). Concretamente, en Andalucía la situación de desempleo juvenil la colocan en la lista de regiones europeas en peores condiciones $(53,71 \%$ de los 16 a los 19 años y $38,01 \%$ de los 20 a los 24 años).

Asimismo, el número de población de migrantes no acompañados que llegan a las costas andaluzas e ingresan en el Sistema de Protección de Menores es muy elevado: 7.783 nuevos ingresos en 2018 según el
Observatorio de la Infancia en Andalucía (2019). Esta situación precisa, que la atención al colectivo de jóvenes extutelados se entienda como un problema social de alta prioridad y de derechos sociales (Del Valle y Bravo, 2013).

Las investigaciones nacionales $\mathrm{e}$ internacionales sobre la transición a la vida adulta de jóvenes extutelados han aumentado en los años recientes (Rutman y Hubberstey, 2016; Courtney y Hook, 2017; Heerde, Hemphill y Scholes-Balog, 2018; Gradaílle, Montserrat y Ballester, 2018), evidenciando la importancia de esta cuestión, aunque hay muchas discrepancias entre los diferentes países europeos sobre los marcos normativos y los recursos disponibles (Del Valle y Bravo, 2013).

El panorama europeo es muy diverso. El Reino Unido, reformó su legislación en el año 2000 (Children Leaving Care Act), para ampliar el apoyo a estos jóvenes de los 18 a los 21 años (López, et al., 2013). En Alemania, se ha aumentado la edad de los servicios de protección hasta los 21 años (Del Valle y Bravo, 2013). En Noruega, los legisladores han acordado que este grupo necesita una atención especial, aunque el sistema tiene grandes dificultades para ofrecer apoyos a la transición. En Suecia, aunque no existe un respaldo legal, la transición es más exitosa, debido a una mayor disponibilidad de recursos y programas (Storø, Sjöblom y Höjer, 2019).

En España, la Ley Orgánica 26/2015 de modificación del sistema de protección a la infancia y a la adolescencia (Gobierno de España, Ministerio de la Presidencia, 2015), tuvo en cuenta por primera vez medidas para fomentar los procesos de emancipación. Sin embargo, la implementación de políticas específicas y la dotación de recursos depende de las regiones, existiendo muchas diferencias entre ellas, debido a las distintas políticas, a las limitaciones presupuestarias y a las desigualdades económicas entre las regiones.

No obstante, la mayoría de los programas de apoyo a la transción a la vida adulta llevados a cabo en España centran sus esfuerzos en la educación, la preparación para el mercado y las habilidades básicas para la vida independiente, 
mientras que se presta menos atención al apoyo socioemocional (Cassarino-Perez, Ermel y Castellà, 2018). Además, no todos los programas son evaluados rigurosamente, por lo que se desconoce el impacto real que tienen estos (Hadley, Mbwana y Hair, 2010).

El estudio que a continuación se presenta ha sido realizado en el contexto español. El objeto de la investigación, es el desarrollo de la empleabilidad de los jóvenes extutelados y el objetivo principal, por tanto, es conocer los beneficios del programa para facilitar la inserción sociolaboral de los jóvenes en riesgo de exclusión social que proceden del Sistema de Protección, a través de las percepciones de los propios jóvenes y sus orientadores. Este programa, abarca todos los aspectos de la vida cotidiana de los jóvenes que participan en él. No obstante, este estudio se centra concretamente en la orientación laboral y en la inserción de estos jóvenes en el mercado de trabajo.

\section{Jóvenes extutelados y riesgo de exclusión social}

Una causa de la exclusión social de algunos jóvenes puede ser, como indican Lebrero, Montoya y Quintana (2008), la exteriorización y perpetuación de la inadaptación social en la etapa adolescente, a través de actividades delictivas o predelictivas y del consumo de drogas. Estas actividades generalmente están causadas por circunstancias sociales de marginación, conflictividad familiar y social, así como problemas psicológicos.

Los jóvenes procedentes del Sistema de Protección suelen tener algunos de los siguientes perfiles: Poseer niveles bajos de logro educativo y de empleo, sufrir más probabilidades de vivir experiencias de pobreza e inestabilidad de vivienda, convertirse en padres jóvenes, depender de la asistencia social, tener malas o nulas relaciones con sus familiares, estar desempleados o subempleados, abandonar pronto la escuela, ser encarcelados, tener problemas de salud mental y de abuso de sustancias (Reid, 2007; Courtney, 2009; Osgood, Foster y Courtney, 2010; Muller-Ravett y Jacobs, 2012; López et al., 2013; Escarbajal e Izquierdo, 2013; Cosner, Singer y Hokanson, 2014; Peters, Sherraden y Kuchinski, 2016; Heerde, et al., 2018).

Rodríguez, De-Juanas y González (2016), afirman que los jóvenes en situación de vulnerabilidad creen que los beneficios de la formación son poco relevantes. Por ello, tienen más interés en abandonar la formación prematuramente para buscar trabajo, respecto de los jóvenes que no se encuentran en la misma situación. Además, valoran la formación de una manera estratégica, como requisito necesario para tener más oportunidades de acceder a empleos con mejores condiciones. Los jóvenes saben que sin una formación mínima (en el caso español, el Graduado en Educación Secundaria Obligatoria, que se obtiene a los 16 años) no pueden acceder al mercado laboral.

La inserción laboral es valorada porque permite obtener ingresos económicos, prestar ayuda económica a las familias y afrontar el proceso de emancipación. No obstante, los jóvenes extutelados tienen menos posibilidades de alcanzar estudios secundarios (Courtney, 2009; Courtney y Hook, 2017). En este sentido, Zúñiga y Soriano (2019) demuestran que los programas de inserción laboral para jóvenes en Andalucía tienen menor impacto en aquellos que tienen una baja cualificación $y$, por tanto, presentan mayores dificultades de inserción laboral.

A pesar de estas dificultades, según Osgood, et al. (2010), muchos jóvenes consiguen el nivel básico de autosuficiencia. Los jóvenes que consiguen tener éxito suelen caracterizarse por poseer temple, capacidad para superar las dificultades y habilidades para recuperarse rápidamente de eventos estresantes o traumáticos. Otros factores que favorecen una transición exitosa a la vida adulta son: El apoyo de la familia y de los amigos; rasgos de personalidad como la persistencia y la confianza; y los buenos resultados escolares, que tienen relación con el acceso al empleo (Osgood, et al., 2010; Chávez y Peralta, 2019). 


\section{El Programa Mayoría de Edad +18: Facilitando la transición a la vida adulta desde la práctica}

De acuerdo con Zúñiga y Soriano (2019), las políticas de empleo juvenil deberían de estar ajustadas a los jóvenes que tienen mayores dificultades para integrarse en el mercado de trabajo y deben diseñarse programas que se ajusten a las necesidades y vulnerabilidades de cada grupo de joven. En esta misma línea, Castro, García y López (2020), plantean que la inclusión social juvenil no supone solo implantar estrategias laborales, sino que, además, es primordial atender a las necesidades individuales, valía personal, sociales y de inclusión ciudadana.

El Programa Mayoría de Edad +18 pretende guiar a jóvenes extutelados, una vez cumplidos los dieciocho años, en el proceso de construcción de su proyecto personal de vida, de manera que logren la plena integración social y laboral, y vivan de forma normalizada y autónoma. El programa abarca numerosos objetivos, referidos a los siguientes aspectos: Ámbito personal, salud e higiene, vivienda, actividades de la vida cotidiana, recursos familiares y comunitarios, formación $\mathrm{y}$ desarrollo laboral.

Por tanto, responde a las tres necesidades de la orientación para la vida adulta según Álvarez (1999): Personal o vocacional, educativa y social. Además, está destinado a jóvenes con perfiles diversos y en los que influyen aspectos tales como la situación personal, la experiencia en su desarrollo personal y las dificultades del núcleo familiar, que siguen estando sin recursos tras salir de los Centros de Protección y que, como consecuencia, entran en una potencial situación de marginación y exclusión social (Rodríguez, 2006).

El Programa Mayoría de Edad +18 fue iniciado en 1997 y se presenta bajo dos formatos, de alta o de media intensidad, en relación con los recursos que ofrece. El subprograma de alta intensidad, se dirige a los jóvenes que son atendidos en diferentes facetas y a los que se les ofrece, además, alojamiento y manutención durante un año, máximo dos, para que alcancen una situación de vida independiente. Por su parte, el subprograma de media intensidad, oferta un proceso de tutorización y acompañamiento externo. Los jóvenes, necesitan volver a estrechar lazos familiares tras salir del Sistema de Protección, pero se dan cuenta de que sus familias no les pueden ofrecer ayuda para iniciar su vida autónoma, lo cual les motiva a participar en el programa de forma voluntaria.

El proceso de incorporación al programa es básicamente informativo y se realiza por parte de los profesionales, en sesiones informativas de grupo o individuales, en las que se les ofrece toda la información que necesitan conocer para poder decidir la integración en el programa. Por otro lado, se entrevista a los educadores de los jóvenes de los Centros de Protección y se desarrolla una coordinación continuada con los profesionales del área de Asuntos Sociales e Igualdad de la administración pública.

La asociación en la que se centra la presente investigación, denominada bajo el nombre ficticio Orienta, tiene un abanico amplio de servicios centrados en discapacidad intelectual, menores tutelados, cooperación internacional y empleo. Entre ellos se destacan: Los Centros de Protección a la Infancia (Centros Residenciales, Centro Terapéutico y Centro de Atención Inmediata); los diferentes programas (Mayoría de Edad +18 , Pre-mayoría y otros programas destinados a personas con discapacidad intelectual); y otros servicios destinados a personas adultas con discapacidad (servicios de día, unidad de estancia diurna y servicios residenciales).

El Programa Mayoría de Edad +18 comenzó a funcionar en la asociación Orienta en el año 2003, y desde entonces ha contado con dos apartamentos, uno para chicos y otro para chicas, dos orientadores sociolaborales y un asesor laboral. En este recurso de Alta Intensidad se trabajan tres áreas, relativas a dimensiones personales, sociales y laborales. Cada área tiene varios objetivos específicos, que complementan al objetivo general. Las áreas personal y social, se trabajan diariamente 
con los jóvenes, colocándolas en práctica desde la vida cotidiana, desmontando falsas expectativas y favoreciendo la aceptación gradual de las propias capacidades. El área laboral se trabaja de forma individual, enfatizando la necesidad de mantener un puesto de trabajo y motivando la flexibilidad sobre las expectativas.

\section{Metodología}

El objetivo principal de esta investigación es conocer los beneficios del Programa de Mayoría de Edad +18 para facilitar la inserción sociolaboral de los jóvenes en riesgo de exclusión social que proceden del Sistema de Protección. En ese sentido, al tratarse de un estudio exploratorio, se utiliza la investigación descriptiva con una metodología mixta, combinando enfoques cualitativos $\mathrm{y}$ cuantitativos. El diseño de la metodología mixta es fijo y emergente; es decir, antes de iniciar la investigación se planifica el uso de la metodología cuantitativa y cualitativa, en dos fases distintas (Creswell y Plano, 2011), aunque interdependientes una fase de la otra.

\subsection{Población y muestra}

La población objeto de investigación son los jóvenes extutelados que abandonan el Sistema de Protección y acceden al Programa de Mayoría de Edad +18 , en Andalucía (España). La muestra seleccionada estuvo conformada por los dos orientadores que forman parte del programa y un total de 10 jóvenes de una entidad de la provincia de Sevilla. La selección de la muestra se obtuvo mediante el muestreo no probabilístico, definido como el proceso de selección que no depende de la probabilidad (Bisquerra, 2014). Se eligió este tipo de muestreo por ser una población conocida y de fácil acceso.

En el caso de los orientadores, ambos son profesionales de la educación con 9 años de experiencia en el programa. Concretamente, una pedagoga y un maestro. En el caso de los jóvenes, 6 de los participantes seguían siendo beneficiarios del programa, mientras que los otros 4 participantes habían pasado por él y lo habían finalizado, por lo que se consideran egresados (ver Tabla 1).

Tabla 1.

Características de los jóvenes participantes en la investigación

\begin{tabular}{lccl}
\hline \multicolumn{1}{c}{ Sexo } & Edad & Estancia en el programa & \multicolumn{1}{c}{ Carácter } \\
\hline Mujer & 18 & $0-6$ meses & Beneficiario \\
Mujer & 18 & $0-6$ meses & Beneficiario \\
Mujer & 19 & $13-18$ meses & Beneficiario \\
Mujer & 19 & $13-18$ meses & Beneficiario \\
Hombre & 18 & $0-6$ meses & Beneficiario \\
Hombre & 19 & $19-24$ meses & Beneficiario \\
Mujer & 19 & $0-12$ meses & Egresado \\
Mujer & 20 & $0-12$ meses & Egresado \\
Hombre & 20 & $13-24$ meses & Egresado \\
Hombre & 23 & $13-24$ meses & Egresado \\
\hline
\end{tabular}

Fuente: Elaboración propia, 2020. 
Cabe señalar, que todos los participantes fueron informados de la naturaleza de la investigación, y de la utilización de los datos únicamente con fines científicos. Su participación fue voluntaria, y se informó que se mantendría la confidencialidad así como el anonimato para salvaguardar la identidad. Durante la fase de recogida de datos se utilizaron seudónimos para proteger el anonimato de los participantes.

\subsection{Instrumentos}

Los instrumentos de recogida de datos fueron diversos. En el caso de los orientadores, se realizaron entrevistas semiestructuradas a partir de un guion con 9 cuestiones. En este sentido, se considera que las entrevistas eran un apoyo eficaz a otras técnicas de investigación cualitativa, puesto que "son el instrumento más poderoso de una investigación, cuando se las utiliza en conjunción con otros métodos" (Woods, 2011, p.104).

A los jóvenes beneficiarios del programa, se les realizó un cuestionario ad hoc de escala tipo Likert, con 17 items, mientras que a los jóvenes egresados del programa, se les aplicó también un cuestionario ad hoc tipo Likert, pero con 24 items. Ambos instrumentos constaban de los siguientes bloques: Valoración sobre el programa, los orientadores, las expectativas, la transición a la vida adulta y la inserción sociolaboral. La validez del contenido de ambos cuestionarios fue valorada por 10 expertos, lo que supuso la modificación de algunos ítems y la eliminación de otros, hasta su versión final. En el caso de los jóvenes, se optó por la técnica de la encuesta, debido a que los sujetos investigados proporcionan información relevante sobre sí mismos y/o su entorno (Bisquerra, 2014).

\subsection{Análisis de los datos}

El procedimiento de análisis utilizado sigue los pasos que proponen Hernández, Fernández y Baptista (2014) y parte de la teoría cimentada, pues "la teoría (hallazgos) va emergiendo fundamentada en los datos" (p.444). Con el objetivo de documentar todo el proceso analítico, así como de aportar validez y confiabilidad al estudio, se usó la bitácora de análisis.

En el caso de los datos recogidos de los orientadores, una vez transcritas las entrevistas, se revisaron todos los materiales obtenidos y se organizaron los datos mediante el análisis temático de los datos cualitativos. Para ello, se identificaron análisis de significados, se organizaron partiendo de categorías y códigos preestablecidos o bien añadiendo nuevas categorías o códigos emergentes. Posteriormente, se definieron relaciones entre los segmentos textuales, para identificar diferencias y similitudes.

En un segundo plano de análisis, se compararon las categorías y se definieron cada una de ellas por temáticas. En este proceso, se describieron las categorías, se les dio significado, se vio la presencia de cada una de las categorías según la frecuencia de aparición en los materiales analizados y se encontraron vinculaciones o relaciones entre cada una de las categorías. La frecuencia de los datos cualitativos, resulta del conteo del total de las categorías analizadas de la técnica de entrevista a través del programa de análisis Aquad 7.

En el caso de los detalles recogidos de los jóvenes, el análisis de los datos cuantitativos se realizó mediante el programa cuantitativo SPSS v. 26. En primer lugar, se volcó la matriz de datos en el programa y se revisaron las variables de investigación. Posteriormente, se realizó un estudio descriptivo de los porcentajes y frecuencias de los items del cuestionario que corresponden a las categorías estudiadas. Al respecto, se establecieron 9 dimensiones de análisis, sin embargo, en este artículo se abordará solo tres dimensiones por las relaciones que existen entre ellas (ver Figura I): Seguimiento/acompañamiento, expectativas y percepciones. 


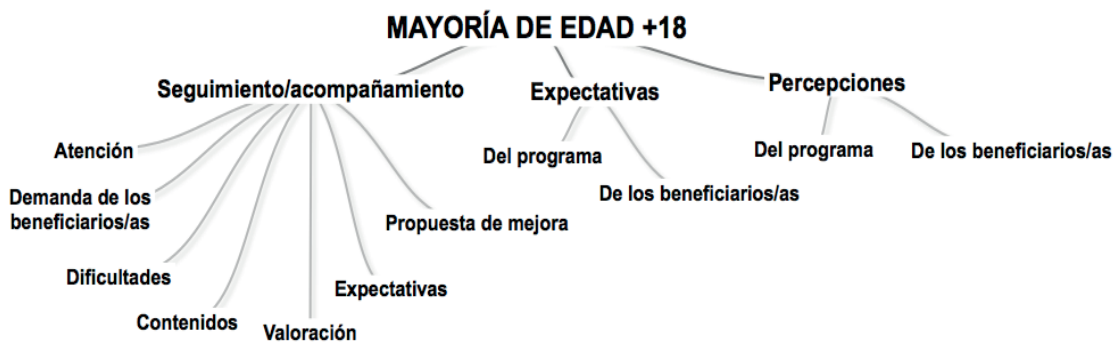

Fuente: Elaboración propia, 2020.

Figura I: Árbol del sistema de categorías

\section{Resultados y discusión}

A continuación, se presentan los resultados obtenidos tras el análisis cuantitativo y cualitativo de las dos técnicas de investigación usadas, colocando el foco en las categorías seleccionadas.

\subsection{El seguimiento y el acompañamiento como valor fundamental}

La totalidad de los jóvenes beneficiarios, consideraron que los orientadores les estaban ayudando a tomar decisiones que favorecían su futuro laboral y que estaban bien formados para llevar a cabo la labor que realizan. El $66,7 \%$ se sienten cómodos hablando con los orientadores y consideran que estos los escuchan cuando expresan sus necesidades.

Del mismo modo, todos los egresados estaban de acuerdo con los items de esta dimensión. Tan solo el $25 \%$ de estos sujetos estaban de acuerdo con que se sentían cómodos hablando con los orientadores, y no estaban ni en acuerdo ni en desacuerdo con que los orientadores estuvieran bien formados para realizar su trabajo. También hay que añadir que el $50 \%$ de este grupo estuvo en desacuerdo con la afirmación de que haber participado en el programa haya acortado el periodo de desempleo.

Sin embargo, todos estuvieron de acuerdo en que los orientadores les habían ayudado a tomar decisiones que habían favorecido su situación laboral. López, et al. (2013), ya enfatizaban la importancia de los educadores como figuras de apoyo y la necesidad de que sean percibidos positivamente por los beneficiarios. A continuación, se muestra en el Cuadro 1 los resultados de las entrevistas a los orientadores:

\section{Cuadro 1}

\section{El seguimiento y el acompañamiento según los orientadores}

\begin{tabular}{lll}
\multicolumn{1}{c}{ Subcategoría } & Interpretación & \multicolumn{1}{c}{ Declaraciones de apoyo } \\
\hline $\begin{array}{l}\text { Dificultades en el } \\
\text { proceso }\end{array}$ & $\begin{array}{l}\text { Por los propios jóvenes } \\
\text { u otras cuestiones }\end{array}$ & $\begin{array}{l}\text { "tampoco muchas veces tienen claro dónde quieren trabajar, no tienen } \\
\text { experiencia laboral prácticamente ninguno... Entonces hay que trabajar } \\
\text { prácticamente desde cero con ellos. Básicamente desde el principio con } \\
\text { ellos" (Profesional A) }\end{array}$
\end{tabular}




\section{Cont... Cuadro 1}

\begin{tabular}{|c|c|c|}
\hline Atención ofrecida & $\begin{array}{l}\text { Individualizada, } \\
\text { personalizada adaptada } \\
\text { a las necesidades y } \\
\text { situación concreta del } \\
\text { joven }\end{array}$ & $\begin{array}{l}\text { "[Pone el ejemplo de un chico, mencionando su nombre]... Pues yo si } \\
\text { tengo que hacer un seguimiento un poquito más del día a día, pues se } \\
\text { le hace; yo creo que se intenta que el seguimiento de cada chaval sea el } \\
\text { justo para él" (Profesional A) }\end{array}$ \\
\hline $\begin{array}{l}\text { Propuestas } \\
\text { mejora }\end{array}$ & $\begin{array}{l}\text { Mayor apoyo de la } \\
\text { Administración/otros } \\
\text { profesionales }\end{array}$ & $\begin{array}{l}\text { "Cuando salen, quizás recoger ese seguimiento externo, ese } \\
\text { seguimiento que tenemos o esas ayudas puntuales que hacemos con los } \\
\text { chicos que han salido ya del programa" (Profesional B) }\end{array}$ \\
\hline $\begin{array}{l}\text { Demanda de } \\
\text { beneficiarios }\end{array}$ & $\begin{array}{l}\text { Mayor } \\
\text { acompañamiento y } \\
\text { atención }\end{array}$ & $\begin{array}{l}\text { "Sí que es verdad, que demandan, sobre todo las chicas, que yo me } \\
\text { centro más en las chicas, demandan mi atención, que algunas veces es } \\
\text { bueno y otras veces no porque se crea un poco más de dependencia. } \\
\text { Y nuestro trabajo precisamente consiste en que sean un poco más } \\
\text { autónomos, que sean más independientes" (Profesional B) }\end{array}$ \\
\hline $\begin{array}{l}\text { Valoración de sus } \\
\text { funciones }\end{array}$ & $\begin{array}{l}\text { Acompañamiento } \\
\text { adecuado para } \\
\text { conseguir la autonomía }\end{array}$ & $\begin{array}{l}\text { "Yo creo que ya más sería contraproducente. ¿Por qué? Porque } \\
\text { nuestros chavales yo quiero que sean los más autónomos posibles." } \\
\text { (Profesional A) }\end{array}$ \\
\hline Contenidos & Ámbito laboral y social & $\begin{array}{l}\text { "Desde el currículum, de la preparación del currículum, preparación de } \\
\text { entrevistas, simulación de entrevistas, el tema de las habilidades pre- } \\
\text { laborales, tema de prácticas..." (Profesional B) }\end{array}$ \\
\hline $\begin{array}{l}\text { Trabajo sobre las } \\
\text { expectativas }\end{array}$ & $\begin{array}{l}\text { Entrevista inicial } \\
\text { y trabajar desde la } \\
\text { realidad }\end{array}$ & $\begin{array}{l}\text { "Para mí la base es la realidad, que conozcan su realidad cuál es y } \\
\text { a partir de ella, una vez que la han aceptado, empezar a trabajar" } \\
\text { (Profesional A) }\end{array}$ \\
\hline
\end{tabular}

Fuente: Elaboración propia, 2020.

Los orientadores, encuentran muchos obstáculos para realizar el seguimiento y acompañamiento de los jóvenes. Principalmente, estas dificultades están relacionadas con los propios jóvenes, sus intereses (estudiar o trabajar), la procedencia geográfica y las carencias en el desarrollo madurativo y emocional, debido a las experiencias difíciles que han vivido. En otras ocasiones, estos problemas se refieren a otras cuestiones: El tiempo limitado con que cuentan los orientadores para trabajar con los jóvenes, pues cuando pueden profundizar en el ámbito laboral el programa finaliza; la necesidad del apoyo externo de la Administración, para canalizar la ayuda que estos jóvenes necesitan; o la dificultad de mantener una empresa colaboradora.
La atención que reciben los jóvenes es individualizada, teniendo en cuenta sus necesidades concretas, pues estos se encuentran en diferentes puntos de partida. Esto supone, que la orientación se concibe como un proceso personalizado. Por tanto, a la hora de trabajar el contenido laboral con los jóvenes, se tiene en cuenta la situación específica de estos, dependiendo de sus conocimientos, su grado de autonomía y otros factores. Este seguimiento individualizado y personal, se realiza a través de tutorías cada cierto tiempo, o bien mediante el móvil, de acuerdo con el perfil de cada uno. Según Martínez (2010), esta es la forma en que debe de ser atendida esta población en los programas de orientación profesional, para que desarrollen el conocimiento del entorno y de ellos mismos, de manera que sean capaces de 
tomar decisiones sobre su futuro profesional.

Por otro lado, los orientadores colocan el énfasis en el escaso apoyo de la Administración, es decir, consideran que deberían de recibir más colaboración de las autoridades públicas, con el fin de ayudar mejor a los jóvenes. Los orientadores también proponen: Desarrollo de un trabajo personal con los jóvenes, en relación al estado psicológico en el que se encuentran, como base para trabajar otros ámbitos; mejorar el acondicionamiento de las viviendas; puntos de encuentro de las personas que trabajan en programas de Mayoría de Edad de ámbito provincial; más recursos personales; creación de una red de empresas actualizada; incidir más con algunos jóvenes en los contenidos y en la orientación laboral; y registrar de forma sistemática el seguimiento que se realiza a los jóvenes que acaban el programa.

Los jóvenes beneficiarios del programa, proponen como mejoras la incorporación de un psicólogo y disponer de conexión Wifi. Igualmente, los jóvenes egresados comentan que mejorarían el programa introduciendo Internet con la finalidad de facilitar el estudio y aumentando la atención así como la observación por parte de los profesionales.

Los orientadores, consideran que los jóvenes demandan mayor acompañamiento y mucha atención para cubrir sus necesidades afectivas a través de los propios orientadores o de las parejas. Sin embargo, opinan que el acompañamiento que llevan a cabo es el justo y necesario, teniendo en cuenta que el objetivo es que los jóvenes trabajen la autonomía y sean más independientes. Además, uno de los orientadores consideró que el modo de actuar en el programa es similar al de una empresa de inserción (proceso individualizado de formación, orientación y búsqueda de trabajo adaptada a necesidades). Por este motivo, este orientador considera que contar con empresas de inserción como recurso, puede suponer un retroceso en el proceso de inserción de los jóvenes atendidos en el programa.

De igual manera, los orientadores explican que se trabajan contenidos referidos a cómo enfrentarse a una entrevista de trabajo, la preparación del currículum, la realización de prácticas y las habilidades pre-laborales. En el ámbito social, también se trabaja la realización de gestiones y el conocimiento sobre los recursos disponibles, la zona en la que viven, así como los derechos y deberes.

En cuanto al seguimiento de las expectativas, lo primero que los orientadores hacen es entrevistar a los jóvenes al inicio del programa, para conocer sus intenciones, metas y perspectivas. Además, los orientadores son realistas cuando trabajan las expectativas de los jóvenes, para que estos conozcan su realidad y la acepten, como base para iniciar el trabajo con ellos y ellas.

\subsection{Las percepciones y las expectativas de los participantes}

Dentro de la dimensión "Percepciones" hay que destacar que el $100 \%$ de los beneficiarios se consideran privilegiados por tener la oportunidad de participar en el programa. Osgood, et al. (2010) ya hacían referencia a cómo las políticas públicas presentaban problemas en cuanto al número de plazas ofertadas, debido a la posible exclusión de jóvenes con el perfil adecuado para ser beneficiarios. No obstante, este problema puede ocasionar dos situaciones distintas. Por un lado, el joven seleccionado para participar en el programa puede considerarse favorecido con respecto al resto de jóvenes, y considerar esto como una oportunidad. Esta situación puede traer como consecuencia, una mayor motivación del joven a la hora de implicarse en las acciones del programa. Por otro lado, el hecho de dejar fuera a potenciales beneficiarios limita el impacto del programa y favorece el aumento de comportamientos antisociales.

Todos los jóvenes consideraron igualmente, que es necesario continuar siendo orientados en los distintos aspectos trabajados durante el programa. Al respecto, Osgood, et al. (2010) explicaba que la independencia es la meta de estos jóvenes y que sus posibilidades de éxito aumentan gracias a los apoyos que reciben, para aumentar poco a poco el control de sus propias vidas, con la ayuda de los 
orientadores.

E1 25\% de los jóvenes estaba totalmente en desacuerdo con que estuvieran incorporados al mercado laboral y tuvieran empleo, que el programa les hubiera ayudado en su inserción laboral y que les quedase mucho por hacer para conseguir un empleo acorde con sus metas y expectativas profesionales. Aunque, por otra parte, el $50 \%$ está en desacuerdo con que le quede mucho camino para lograr un empleo en el que se sientan realizados. No obstante, esto puede deberse a motivos externos al programa, por la crisis económica y la falta de ofertas de empleo, pudiendo restar impacto por la posibilidad de pérdida de motivación e implicación. En cualquier caso, como recuerdan López, et al. (2013) y Martín (2015), los jóvenes procedentes del Sistema de Protección presentan más dificultades para encontrar un empleo.

En cuanto a las "Percepciones" específicas del programa, los orientadores consideran que el programa es totalmente necesario y que el trabajo realizado con los jóvenes es muy positivo. El orientador, comentó que el programa se conoce poco y que esto constituye un error de los servicios sociales. La orientadora, por su parte, consideró que tienen poco tiempo para realizar sus funciones, puesto que los orientadores deben de atender otras cuestiones dentro de la asociación, además del programa. Por ello, la orientadora manifestó que le gustaría dedicar más tiempo y más atención a los jóvenes. "De hecho, si no existiera el programa de Mayoría de Edad como recurso tras la protección, sería absurdo seguir tutelando a los chavales. Sería ilógico" (Profesional B).

Por otra parte, respecto a las "Percepciones" de los beneficiarios, el orientador comenta que los jóvenes se lamentan de la falta de continuidad de la tutela, una vez cumplidos los dieciocho años. En efecto, no entienden cómo al alcanzar la mayoría de edad son devueltos al entorno que provocó la tutela, sobre todo sin haber solucionado el problema familiar o social que hizo necesaria la misma.

Asimismo, el 66,7\% de los beneficiarios considera que tiene altas expectativas de encontrar un empleo, que el programa va a ayudar a dar respuesta a sus perspectivas laborales y que, tras haber realizado el programa, tendrán más posibilidades de encontrar trabajo. Sin embargo, un 16,7\% están en desacuerdo con que su formación sea adecuada para conseguir un empleo.

No obstante, los egresados, a diferencia de los jóvenes beneficiarios del programa, tienen diferentes perspectivas sobre las expectativas de encontrar un empleo. El $75 \%$ de los egresados estuvieron totalmente de acuerdo en que el programa les había ayudado a dar respuestas a las mismas, y el $25 \%$ estuvieron totalmente en desacuerdo con que el programa ayudara a aumentar las expectativas laborales.

En las entrevistas a los orientadores se pudo explorar las expectativas de los beneficiarios y las del programa. Por un lado, las de los beneficiarios suelen estar referidas, según los orientadores, a encontrar un trabajo, ganar un buen sueldo, independizarse y ser autónomos. "Claro, las expectativas son eso. Ellos creen que van a salir, desde que llegan a Mayoría van a ganar 1.500, que van a encontrar una casa rápido, y eso no suele pasar" (Profesional A). Por otro lado, respecto a las expectativas del programa, los orientadores consideran que estas se cumplen.

\section{Conclusiones}

Con este estudio se evidencia que los programas de transición a la vida adulta son de vital importancia, especialmente para los jóvenes extutelados, debido a que esta etapa es cada vez más larga y difícil, y a que sus efectos negativos aumentan en jóvenes que llevan gran parte de su vida viviendo bajo la tutela del Sistema de Protección de Menores.

En primer lugar, el programa es visto de manera positiva por los agentes participantes, aunque los orientadores hacen hincapié en el escaso apoyo que reciben por parte de la Administración y de las ONG. Esta cuestión da a entender un relativo sentimiento de soledad a la hora de realizar el trabajo de orientación. 
En segundo lugar, tanto los jóvenes beneficiarios como los egresados se sienten muy privilegiados por participar en el programa, pues supone una oportunidad única. Los jóvenes resaltan el beneficio de poder participar en el programa, dado que muchos otros jóvenes no pueden conseguir una plaza al ser estas limitadas.

Por su parte, los orientadores consideran que los jóvenes presentan un alto grado de inmadurez, lo que provoca que estos demanden más atención y seguimiento del que suelen recibir. En este sentido, los jóvenes tienen carencias emocionales, que difícilmente pueden cubrirse desde el programa. Además, existen limitaciones en la inserción laboral, pues debido a la corta duración del programa no se puede trabajar en profundidad la transición hacia el empleo.

En tercer lugar, tanto los orientadores como los beneficiarios y egresados, piensan que el programa es muy necesario y que el trabajo que desarrollan en él es muy positivo, aunque ni el programa ni la labor que este realiza son conocidos. La falta de difusión, implica el desconocimiento de potenciales usuarios. Además, provoca que no se valoren los beneficios sociales del programa. Un mejor conocimiento de este por parte de la opinión pública, podría favorecer una mayor inversión en este tipo de servicios sociales y también repercutirían en las oportunidades laborales de estos jóvenes.

Con todo esto, se considera que el objetivo principal del estudio (describir e interpretar, desde la perspectiva de los participantes, los posibles beneficios del programa con foco en la inserción sociolaboral de los jóvenes procedentes del Sistema de Protección que están en riesgo de exclusión social) queda suficientemente clarificado. En efecto, el estudio confirma que tanto los jóvenes como los orientadores, opinan que el programa les ayuda significativamente en la inserción laboral de los usuarios. Además, el programa demuestra la gran utilidad para dar continuidad a los años de tutela, siendo el último recurso para trabajar las competencias sobre autonomía, independencia y factores personales así como emocionales.

Finalmente, de forma general, teniendo en cuenta tanto las perspectivas de los participantes como la literatura revisada, se sugiere como posibles mejoras las siguientes: El aumento de la implicación de la Administración pública, tanto con los jóvenes que tutela como con los orientadores, educadores y otros profesionales, para que reciban todos los apoyos necesarios; las ONG deben de desempeñar un papel más importante y protagonista en el desarrollo de programas de transición a la vida adulta de los jóvenes extutelados. Esto supone favorece un mayor asesoramiento y un mejor acceso a los recursos disponibles; y, por último, otra propuesta de mejora del programa de Mayoría de Edad +18 , consiste en la creación de un registro del seguimiento que se hace a los jóvenes salientes del programa, con la finalidad que quede constancia de cómo y con qué frecuencia reciben ayuda.

Los Centros de Menores, deben de contribuir a concienciar socialmente sobre la importancia del programa de transición, para evitar las numerosas bajas que se producen. Por su parte, la Administración debería de favorecer encuentros frecuentes entre los profesionales dedicados a los programas de transición. Asimismo, es importante desarrollar programas de atención a las familias en riesgo de exclusión, pues constituyen el origen de las situaciones que llevan a estos jóvenes a ser tutelados.

No se debe olvidar, que este proceso de inserción y acompañamiento debe tener como objetivo central la mejora de las condiciones de vida de los jóvenes en riesgo de exclusión, facilitando así la mejora y el desarrollo de las personas y, por ende, del tejido social y de la ciudadanía.

En ese sentido, futuras investigaciones deberían ir encaminadas a conocer cuál es el trabajo de preparación para la vida adulta e independiente que se realiza desde los Centros de Protección de menores en materia de formación, autonomía, inserción laboral y atención a aspectos emocionales. 


\section{Referencias bibliográficas}

Álvarez, M. (1999). Orientación profesional. Cedecs.

Bisquerra, R. (Coord.) (2014). Metodología de la investigación educativa. La Muralla, S. A.

Cassarino-Perez, L., Ermel, V., Montserrat, C., y Castellà, J. (2018). Transition from care to adulthood: A systematic review of intervention programs. Trends in Psychology, 26(3), 1665-1681. https:// doi.org/10.9788/tp2018.3-19pt

Castro, L. K., García, C. H., y López, R. E. (2020). Exclusión social, inclusión política y autoestima de jóvenes en pobreza, Monterrey, México. Revista de Ciencias Sociales (Ve), XXVI(1), 38-50. https://doi.org/10.31876/rcs. v26i1.31309

Chávez, J. R., y Peralta, R. Y. (2019). Estrés académico y autoestima en estudiantes de enfermería, Arequipa-Perú. Revista de Ciencias Sociales (Ve), XXV(E-1), 384-399. https://doi.org/10.31876/rcs. v25i1.29629

Coronel, F. T., y Farje, C. A. (2020). Percepción social para reinserción de privados de libertad en Perú: Efecto del edentulismo. Revista de Ciencias Sociales (Ve), XXVI(2), 236$249 . \quad$ https://doi.org/10.31876/rcs. v26i2.32437

Cosner, S., Singer, E., y Hokanson, K. (2014). Emerging versus emancipating: The transition to adulthood for youth in foster care. Journal of Adolescent Research, 29(5), 616-638. https://doi. org/10.1177/0743558414528977

Courtney, M. E. (2009). The difficult transition to adulthood for foster youth in the US: Implications for the state as corporate parent. Social Policy Report, XXIII(1), 3-19.
Courtney, M. E., y Hook, J. L. (2017). The potential educational benefits of extending foster care to young adults: Findings from a natural experiment. Children and Youth Services Review, 72, 124-132. https://doi.org/10.1016/j. childyouth.2016.09.030

Creswell, J. W., y Plano, V. L. (2011). Designing and conducting mixed methods research. SAGE Publications.

Del Valle, J. F., y Bravo, A. (2013). Current trends, figures and challenges in out of home child care: An international comparative analysis. Psychosocial Intervention, 22(3), 251-257. https:// doi.org/10.5093/in2013a28

Dworsky, A., y Gitlow, E. (2017). Employment outcomes of young parents who age out of foster care. Children and Youth Services Review, 72, 133-140. https://doi.org/10.1016/j. childyouth.2016.09.032

Escarbajal, A., e Izquierdo, T. (2013). Percepciones psicosociales de la exclusión que determinan la inclusión sociolaboral. Revistas de Ciencias Sociales (Ve), XIX(1), 13-21.

Eurostat (2020). European statistical recovery dashboard. Eurostat most recent available data (2018). https:// ec.europa.eu/eurostat/data/database

Gobierno de España. Ministerio de la Presidencia (1978). Constitución Española. Boletín Oficial del Estado, núm. 311, de 29 de diciembre de 1978. https://www.boe.es/buscar/act. php?id=BOE-A-1978-31229

Gobierno de España. Ministerio de la Presidencia (2015). Ley 26/2015, de 28 de julio, de modificación del sistema de protección a la infancia y a la adolescencia. Boletín Oficial del Estado, núm. 180, de 28 de julio de 2015. https://www.boe.es/buscar/act. php?id=BOE-A-2015-8470 
Sevillano-Monje, Verónica; Ballesteros-Moscosio, Miguel-Ángel y González-Monteagudo, José

Programa de transición a la vida adulta en Andalucía: Percepción de profesionales y extutelados

Gradaílle, R., Montserrat, C., y Ballester, L. (2018). Transition to adulthood from foster care in Spain: A biographical approach. Children and Youth Services Review, 89, 54-61. https://doi. org/10.1016/j.childyouth.2018.04.020

Gudžinskienè, V., Raudeliūnaitè, R., y Uscila, R. (2017). The experiences of social workers while preparing children for independent living in the community foster care homes. Pedagogika, 125(1), 158-174. https:// doi.org/10.15823/p.2017.12

Hadley, A. M., Mbwana, K., y Hair, E. C. (2010). What works for older youth during the transition to adulthood: Lessons from experimental evaluations of programs and interventions. Child Trends, (5), 1-15.

Heerde, J. A., Hemphill, S. A., y ScholesBalog, K. E. (2018). The impact of transitional programmes on posttransition outcomes for youth leaving out-of-home care: a meta-analysis. Health and Social Care in the Community, 26(1), e15-e30. https:// doi.org/10.1111/hsc. 12348

Hernández, R., Fernández, C., y Baptista, M. D. P. (2014). Metodología de la investigación. McGraw-Hill.

Instituto Nacional de Estadística - INE (2020). Tasa de paro por distintos grupos de edad, sexo y comunidad autónoma. INE. https://www.ine.es/jaxiT3/Tabla. htm? $\mathrm{t}=4247$

Jurado, P., Olmos, P., y Pérez, A. (2015). Los jóvenes en situación de vulnerabilidad y los programas formativos de transición al mundo del trabajo. Educar, 51(1), 211-224.

Lebrero, M. P, Montoya, J. M., y Quintana, J. M. (2008). Pedagogía social. UNED.

López, M., Santos, I., Bravo, A., y Del Valle, J. F. (2013). El proceso de transición a la vida adulta de jóvenes acogidos en el sistema de protección infantil. Anales de Psicología, 29(1), 187-196. https:// doi.org/10.6018/analesps.29.1.130542

Martín, E. (2015). Niños, niñas y adolescentes en acogimiento residencial. Un análisis en función del género. Revista Qurriculum, 28, 88-102.

Martínez, M. P. (2010). Orientación profesional para la transición. En B. Echeverría, S. Isus, P. Martínez y L. Sarasola y (Coords.), Orientación profesional (pp. 223-299). Editorial UOC.

Ministerio de Sanidad, Consumo y Bienestar Social (2019). Boletín de datos estadísticos de medidas de protección a la infancia: Boletín número 21. Datos 2018. MSCBS, Centro de Publicaciones. http://www. observatoriodelainfancia.mscbs.gob. es/productos/pdf/Boletin provisional Proteccion 21-Borrador.pdf

Muller-Ravett, S., y Jacobs, E. (April, 2012). After foster care and juvenile justice: a preview of the youth villages transitional living evaluation. $M D R C$, Policy Brief. http://www.mdrc.org/ sites/default/files/After $\% 20$ Foster $\% 20$ Care.pdf

Observatorio de la Infancia en Andalucía (2019). Maltrato y protección. Informe OIA 2019. Serie: Estado de la infancia y adolescencia en Andalucía. Junta de Andalucía, Conserjería de Igualdad, Políticas Sociales y Conciliación. https://www.observatoriodelainfancia. es/ficherosoia/documentos/5896 d 2 MALTRATO PROTECCION EIA2019.pdf

Osgood, D. W., Foster, E. M., y Courtney, M. E. (2010). Vulnerable populations and the transition to adulthood. The Future of Children, 20(1), 209-229. https:// doi.org/10.1353/foc. 0.0047

Peters, C. M., Sherraden, M., y Kuchinski, A. M. (2016). From foster care to 
adulthood: The role of income. Journal of Public Child Welfare, 10(1), 39-58. https://doi.org/10.1080/15548732.201 $\underline{5.1090940}$

Reid, C. (2007). The transition from state care to adulthood: International examples of best practices. New Directions for Youth Development, (113), 33-49. https://doi.org/10.1002/yd.200

Rodríguez, A. E., De-Juanas, Á., y González, Á. L. (2016). Atribuciones de los jóvenes en situación de vulnerabilidad social sobre los beneficios del estudio y la inserción laboral. Revista Española de Pedagogía, LXXIV(263), 109-126.

Rodríguez, R. (2006). La integración social y laboral de jóvenes que han estado tutelados y tuteladas por la Junta de Andalucía y han cumplido la mayoría de edad. Revista de Estudios de Juventud, (74), 251-277.

Rutman, D., y Hubberstey, C. (2016). Is anybody there? Informal supports accessed and sought by youth from foster care. Children and Youth Services Review, 63, 21-27. https://doi. org/10.1016/j.childyouth.2016.02.007

Saldaña, C., Polo-Vargas, J. D., GutiérrezCarvajal, O. I., y Madrigal, B. E.
(2020). Bienestar psicológico, estrés y factores psicosociales en trabajadores de instituciones gubernamentales de Jalisco-México. Revista de Ciencias Sociales (Ve), XXVI(1), 25-37. https:// doi.org/10.31876/rcs.v26i1.31308

Stein, M. (2006). Young people aging out of care: The poverty of theory. Children and Youth Services Review, 28(4), 422-434. https://doi.org/10.1016/j. childyouth.2005.05.005

Storø, J., Sjöblom, Y., y Höjer, I. (2019). A comparison of state support for young people leaving care in Norway and Sweden: Differences within comparable welfare systems. Child \& Family Social Work, 24(3), 393-399. https://doi.org/10.1111/cfs. 12471

Woods, P. (2011). La escuela por centro. La etnografia en la investigación educativa. Paidós.

Zúñiga, R. M., y Soriano, E. (2019). Desempleo juvenil y programas empleo en Andalucía: ¿La formación como elemento clave? Revista de Ciencias Sociales (Ve), XXV(4), 22-39. 